\title{
Intravenous Administration of Umbilical Cord Mesenchymal Stromal Cells in Advanced-stage Critical COVID-19: a Case Report
}

\section{Katia Nunes Silva}

São Rafael Hospital

Priscila Pinheiro

Hospital São Rafael: Hospital Sao Rafael

Andre Gobatto

Hospital São Rafael: Hospital Sao Rafael

Rogerio Passos

Hospital São Rafael

\section{Bruno Paredes}

D'Or Institute for Research and Education: Instituto D'Or de Pesquisa e Ensino

\section{Luciana França}

D'Or Institute for Research and Education: Instituto D'Or de Pesquisa e Ensino

\section{Carolina Nonaka}

Hospital São Rafael

\section{Beatriz Barreto-Duarte}

Goncalo Moniz Research Centre: Centro de Pesquisas Goncalo Moniz

\section{Mariana Araújo-Pereira}

Goncalo Moniz Research Centre: Centro de Pesquisas Goncalo Moniz

\section{Rafael dos Santos}

Goncalo Moniz Research Centre: Centro de Pesquisas Goncalo Moniz

\section{Fernanda Cruz}

Federal University of Rio de Janeiro: Universidade Federal do Rio de Janeiro

\section{Gabriele Martins}

Hospital São Rafael: Hospital Sao Rafael

\section{Bruno Andrade}

Goncalo Moniz Research Centre: Centro de Pesquisas Goncalo Moniz

\section{Hugo Castro-Faria-Neto}

Oswaldo Cruz Foundation: Fundacao Oswaldo Cruz

\section{Patricia Rocco}

Federal University of Rio de Janeiro: Universidade Federal do Rio de Janeiro

Bruno Solano de Freitas Souza ( $\sim$ bruno.solano@fiocruz.br) 
Centro de Pesquisas Goncalo Moniz https://orcid.org/0000-0003-3771-0439

\section{Short report}

Keywords: COVID-19, ARDS, advanced-stage, mesenchymal stromal cells, cell therapy, immunomodulation

Posted Date: December 23rd, 2020

DOl: https://doi.org/10.21203/rs.3.rs-132721/v1

License: (a) (i) This work is licensed under a Creative Commons Attribution 4.0 International License. Read Full License 


\section{Abstract}

Background: Coronavirus disease 2019 (COVID-19) associated- severe acute respiratory distress syndrome (ARDS) patients may require prolonged mechanical ventilation, thus resulting in lung fibrosis and high fatality rates. Several therapies have been developed in patients with pneumonia requiring oxygen therapy as well as during the early course of invasive mechanical ventilation. Mesenchymal stromal cells (MSCs) may have a role in controlling the hyperinflammatory response seen in such cases and prevent aggravation or increase/accelerate recovery. While MSC-based therapies have been studied mostly in patients that did not require invasive ventilation or during the first hours of tracheal intubation, to date the potential of MSC therapy to treat advanced-stage of severe/critical COVID-19 cases has not been extensively studied.

Methods: This is a case report of a 30-year-old male patient who presented progressive clinical deterioration of COVID-19 in ICU after 21-day admission and 14 days with invasive mechanical ventilation. The first symptom onset was 35 days before MSC therapy. The patient was treated with allogenic human umbilical cord-derived MSCs [5 x 107 (2 doses 2 days interval)].

Results: No serious adverse events attributed to MSC administration were observed during and after the procedure. Oxygenation ( $\mathrm{PaO} 2 / \mathrm{FiO} 2$ ratio) and the need for vasoactive drugs improved. Chest $\mathrm{CT}$ scan imaging, which showed signs of bilateral and peripheral ground-glass, consolidation as well as fibrosis, improved significantly during the time course of the disease. Patient was discharged 13 days after cell therapy. Cytokine analysis demonstrated modulation of different mediators accompanied by modulation of different cell populations in peripheral blood, including a reduction in inflammatory monocytes, increased frequency of patrolling monocytes, CD4+ lymphocytes and type 2 classical dendritic cells (cDC2).

Conclusion: This study described for the first time the effects of MSC therapy in a patient at late stage COVID-19 associated severe lung injury and fibrosis. Therefore, further clinical trials should be design assessing the efficacy of MSC therapy in ARDS patients undergoing prolonged mechanical ventilation due to COVID-19.

\section{Introduction}

SARS-CoV-2 infections present different phenotypes and clinical presentations [1]. Severe pneumonia and acute respiratory failure occur in a subset of patients that require long-term hospitalization in intensive care units (ICU) and prolonged ventilatory assistance [2]. Patients with severe/critical COVID-19 present a hyperinflammatory and hypercoagulable state that may also compromise multiple organs and systems [3]. Mortality rates reported for COVID-19 patients under mechanical ventilation have varied with age and comorbidities from 19 to $73 \%[4,5]$.

Currently, there is no specific treatment to cure patients with COVID-19 infection and only dexamethasone has been shown to decrease mortality [6]. Despite significant therapeutic advances with increased 
knowledge and definition of standard protocols, critical COVID-19 remains a life-threatening disease and novel therapeutic strategies are urgently needed. Moreover, the pandemics continue to accelerate even in countries where the first wave was effectively controlled at first [7]. In this context, cell-based therapies are promising approaches, especially for severe/critical COVID-19 cases [8].

Mesenchymal stromal cells (MSCs) have been evaluated in compassionate use or clinical trials to treat COVID-19 pneumonia [9-18]. The rationale is to direct the immunomodulatory properties of MSCs to control the hyperinflammatory state and improve respiratory function. There are currently over 40 studies registered in clinicaltrials.gov database and a few published case reports or small case series. Most protocols, however, include patients at early-stage disease or shortly after orotracheal intubation. Little attention, however, has been given to late-stage critical cases of COVID-19, in which extensive damage to the lung has already occurred and a fibrotic scar begins to form. Here we report a case of a 30 years-old patient with advanced-stage critical COVID-19 that was successfully treated with umbilical cord-derived MSCs (UC-MSCs).

\section{Methods}

\section{Case presentation}

A 30 years old male patient with no known comorbidities presented on Jun 6th with myalgia, headache, shortness of breath with moderate efforts at illness day 3, SpO2 > 95\%, and a positive test for SARS-CoV2 by nasopharyngeal RT-PCR. At this point, CT scan showed parenchymal ground glass opacities in up to $25 \%$ of the lung parenchyma. The patient was medicated and discharged, returning at illness day 6 with worsened dyspnea, $\mathrm{SpO}=88 \%$ at ambient air, $>50 \%$ altered lung parenchyma on $\mathrm{CT}$, being classified as severe [19]. He was admitted in the ICU at São Rafael Hospital, Salvador, Brazil, receiving oxygen therapy, bronchodilators, anticoagulant, methylprednisolone $(120 \mathrm{mg} /$ day) and antibiotics (ceftriaxone + azithromycin). The patient was treated with high-flow nasal oxygen, non-rebreathing mask and required pronation to sustain $\mathrm{SpO}_{2}$, but did not respond, progressing with desaturation, respiratory acidosis and septic shock, requiring orotracheal intubation on illness day 15 , being clinically diagnosed with critical COVID-19. Laboratory testing was consistent with cytokine storm, with reduced lymphocyte counts, increased C-Reactive Protein (CRP), D-dimer, lactate dehydrogenase (LDH), fibrinogen and ferritin, along with $\mathrm{PaO}_{2} / \mathrm{FiO}_{2}<200$. The patient required vasoactive drugs to keep mean arterial pressure above $65 \mathrm{mmHg}$. On the following days, serial SARS-CoV-2 RT-PCR results were persistently positive and secondary infections with Stenotrophomonas and Klebsiella pneumoniae were detected in the tracheal aspirate, treated with antibiotics. Tracheostomy was performed on Jun 29th. Patient presented clinical deterioration on illness day 30 and CT imaging demonstrated radiological worsening with an acute respiratory distress syndrome (ARDS) pattern, lesions affecting $>75 \%$ of lung parenchyma and foci of interstitial fibrosis. A cell therapy protocol with UC-MSCs was then applied in a compassionate use basis, following informed consent given by the patient's family. 


\section{UC-MSCs}

MSCs were obtained from the umbilical cord tissue at a cGMP facility at the Center for Biotechnology and Cell Therapy, São Rafael Hospital and cryopreserved at passage 3 in $50 \mathrm{ml}$ of a cryopreservation solution containing Plasmalyte, 3\% human albumin and 5\% dimethyl sulfoxide (DMSO) and stored in cryobags at $<-135^{\circ} \mathrm{C}$. MSC's identity was assessed by flow cytometry (Stemflow Human MSC Analysis kit, BD Biosciences) and in vitro trilineage differentiation assays (StemPro Osteogenesis and Adipogenesis kits, ThermoFisher Scientific) (Figure S1A-E). Genetic stability was evaluated by G-band karyotype, as previously described [20] (Figure S1F). Sterility was evaluated by culture for anaerobic, aerobic bacteria and fungi, endotoxin levels and Mycoplasma test. Potency was evaluated by measuring ID01 mRNA expression by RT-qPCR after stimulation with IFNy (Figure S1A), as described previously [21]. Finally, product hemocompatibility was tested by evaluating tissue factor (CD142) expression by flow cytometry and by performing thromboelastography studies in citrate blood samples obtained from three different donors, as previously described (Figure S1B-E) [22]. Finally, cell viability was checked before cryopreservation, $48 \mathrm{~h}$ after, and at the time of infusion, by flow cytometry with 7AAD (BD Biosciences).

\section{Procedures}

Approximately 30-60 min before the infusions, the patient received $50 \mathrm{mg}$ diphenhydramine to prevent infusion-related allergy. The cells were thawed in a $37^{\circ} \mathrm{C}$ water bath and immediately taken to the patient bedside for intravenous infusion, via gravity, over 30-40 minutes. The patient was followed up by daily clinical evaluations and laboratory testing. Radiological evaluation was performed by serial chest X-rays and CT scans. Additionally, blood samples were collected on d1 (pre-infusion), d3 and d7 for evaluation of cytokines and chemokines by Luminex and immune cell populations by flow cytometry (d1, d3, d7 and d14; antibody information in Table S1). To evaluate the overall profile of biomarkers, an unsupervised hierarchical cluster with luminex and flow cytometry assay values was performed using Ward's method. In this analysis, the dendrograms represent the Euclidean distance (inferring degree of similarity). The values were normalized using Z-score method. To calculate a fold-change, d1 was used as reference.

\section{Results}

UC-MSCs are characterized according to their ability to adhere to plastic, and high expression (>95\%) of CD90, CD105, CD44 and CD73, and low expression ( $<2 \%$ of CD45, CD34, CD117 (Figure S1E) and also by their in vitro adipogenic, osteogenic and chondrogenic differentiation, with a normal karyotype (Figure S1F) [23].

Two intravenous administrations of UC-MSCs (50 million cells/infusion) were performed at days 30 and 32. The infusions were well-tolerated, and no adverse events were observed. Patient showed a rapid improvement in oxygenation, requiring progressively lower levels of vasopressors until hemodynamic stability without vasopressors was achieved 9 days post the UC-MSC infusion. The patient was 
discharged 13 days after UC-MSC infusion. Variations in $\mathrm{PaO}_{2} / \mathrm{FiO}_{2}$, SOFA score, lymphocyte counts, CRP, ferritin, and D-dimer, along with the dynamic changes seen in chest CT are shown (Fig. 1). Control CT Scan showed significant absorption of bilateral pulmonary infiltrate, maintaining only retractable opacities.

In order to evaluate possible mechanisms of action of MSCs in immune cells and soluble mediators, we performed Luminex and flow cytometry analyses. An unsupervised hierarchical analysis was performed with Luminex data, and three clusters of plasma biomarkers were established. On the first and second clusters a slight increase in plasma cytokine levels was observed on $\mathrm{d} 3$, compared to $\mathrm{d} 1$. On $\mathrm{d} 7$, the levels of most cytokines approached the first measured value (Fig. 2A-B). On the third cluster the increase was more accentuated and was maintained at $d 7$ (Fig. 2A). For each cluster we performed an enrichment analysis on the $\mathrm{NCl}$ Nature database. The first was enriched mainly to regulation of transcription and signaling process in lymphocytes. The second was enriched to IL-27, calcium and IL-23 signaling. While the third was enriched to IL-12 signaling events (Fig. 2B). The biomarkers that showed the greatest discrepancies (+-0.4-fold change) at the d3 were IL-2RA, IL-18, IL-6 and M-CSF (Fig. 2C).

Flow cytometry analysis demonstrated that, up to seven days following treatment initiation, classical monocytes $\left(\mathrm{CD} 14^{++} \mathrm{CD} 16^{-}\right)$were enriched in the peripheral blood, whereas on $\mathrm{d} 14$, patrolling monocytes $\left(\mathrm{CD} 14^{+} \mathrm{CD} 16^{++}\right)$were the most prevailing monocyte subpopulation (Fig. 3A). Substantial alterations of chemokine receptors expression over time post-treatment, with an increase on CCR $5^{+}$receptors and decrease of $\mathrm{CCR7}^{+}$(Fig. 3B). Additionally, the degree of monocyte activation was substantially altered following treatment in the inflammatory monocyte subpopulation, where it is possible to observe a decrease in these activated monocytes on $\mathrm{d} 3$ and $\mathrm{d} 7$, with subsequent increase on $\mathrm{d} 14$, where the profile is similar to baseline (Fig. $3 \mathrm{C}$ ). Regarding monocyte polyfunctionality in response to a TLR-4 agonist (LPS, $1 \mu \mathrm{g} / \mathrm{mL}$ ), there was a peak of multiple cytokine producer monocytes on $\mathrm{d} 3$, suggesting higher polyfunctional activity on this period (Fig. 3D-F).

The frequencies of $\mathrm{CD} 4^{+}$and $\mathrm{CD} 8^{+} \mathrm{T}$-cells in peripheral blood presented similar changes over time. In both cases there was observed a slight increase at d3, with progressive reduction at d7 and d14 (Fig. 4A). The evaluation of differential chemokine receptor expression of CCR6 and CXCR3 on circulating CD $4^{+}$ lymphocytes revealed higher frequencies of $\mathrm{CXCR}^{-}{ }^{-} \mathrm{CRR}^{-}$(Th2) subpopulation following treatment (Fig. 4B). In activated cells the profile changes, and CXCR3 ${ }^{+} \mathrm{CCR}^{-}$are more frequent (Fig. $4 \mathrm{C}$ ). Interestingly, the patient exhibited a reduction in naïve $\mathrm{CD} 4^{+} \mathrm{T}$ cells frequencies over time along with increased frequencies of terminally differentiated $C D 4^{+} T$ cells (Fig. 4D). Activated TCD $4^{+}$cells were more terminally differentiated and effector in the first day, comparting with the other days (Fig. 4E). Of note, conventional dendritic cells 2 ( $C D C 2$ ) frequency peaked on day 3 after treatment, returning to basal levels afterwards (Fig. 4E).

\section{Discussion}


MSC-based therapy protocols to treat COVID-19 have been directed mainly to patients with moderate and severe clinical presentations [9-18]. Few numbers of studies included critically ill patients with COVID-19 under invasive mechanical ventilation [24-26]. Although preliminary, published data suggests that critically ill patients who presented benefits with the MSC treatment were successfully extubated after receiving MSCs shortly following intubation [24]. Here we report the case of a patient successfully treated with MSCs 14 days after tracheal intubation and invasive mechanical ventilation, in which time association between MSC infusion and amelioration of clinical, oxygenation and laboratory parameters were clearly observed. Importantly, intravenous administration of UC-MSCs was not associated with serious adverse events. This is particularly important in the context of severe/critical COVID-19, due to a thromboinflammatory state [27].

The initial anti-SARS-CoV-2 response starts with the activation of innate immune cells, which function as antigen presenters and produce type I interferons [28]. As the infection progresses and tissue injury increases, an exacerbated inflammatory response, with high levels of pro-inflammatory mediators is seen [29]. Prolonged exposure to a cytokine storm scenario as an expression of dysregulated immune response, leads to macrophage activation syndrome, induced by IL1 $\beta$ [30], defects in the antigen presentation induced by IL-6, decreased HLA-DR expression in monocytes, CD $4^{+} \mathrm{T}$ cell depletion, rapid spread of the virus and secondary organ dysfunction [31]. Poor innate immune response in severe COVID19 was recently characterized as immune paralysis, resembling some characteristics of bacterial sepsis [32]. Our results demonstrate that, after MSC therapy, monocytes increased HLA-DR expression and showed increased ability to respond to TLR4 ligand stimulation. We also observed a marked increase in the frequency of $\mathrm{CDC2}$, accompanied by a transient increase in serum levels of different cytokines that are involved in antigen presentation, antiviral response and differentiation of effector CD4 ${ }^{+} \mathrm{T}$ cells [33]. Interestingly, one of the upregulated pathways found was IL-27 signaling, and dendritic cell-derived IL-27 has been associated with induction of Treg in lung parenchyma and resolution of immunopathology upon infection with respiratory viruses [34].

After $\mathrm{d} 3$, we observed a reduction in proinflammatory cytokines, and in the subset of naïve $\mathrm{CD} 4^{+} \mathrm{T}$ cells, along with increased frequencies of the terminally differentiated subset with Th2 markers. Finally, after d7, we observed increased frequency of patrolling monocytes, a population involved in the resolution of inflammation and healing [35], which migrates to the lungs, differentiate into CD11 $\mathrm{c}^{+}$, resident lung macrophage and act in a specialized way as effector cells [36]. In addition, patrolling monocytes respond strongly to viruses via TLR7/8-MEK pathway, producing cytokines such as TNFa and IL1 $\beta$, as well as CCL5 and CXCL10 chemokines [37, 38].

\section{Conclusions}

The results of this case report support a potential role for MSC-based therapies not only in the early stages of COVID-19, as has been extensively explored, but also in advance -stages of critical disease facing clinical deterioration. Administration of UC-MSCs at day 30 of illness, and 14 days after orotracheal intubation, was still safe and associated with a significant change in the clinical course. 
Further clinical studies with proper design and sample size are required to confirm the efficacy of MSCbased therapies in advanced-stages of severe/critical COVID-19.

\section{List Of Abbreviations}

CT - computed tomography; RT-PCR: Reverse transcription polymerase chain reaction; cGMP - current good manufacturing practices. Remaining abbreviations are cited in the text.

\section{Declarations}

\section{Ethics approval and consent to participate:}

The present study has been approved by the Ethics committee of São Rafael Hospital and CONEP and written consent to participate was given by the patient's family.

\section{Consent for publication:}

Written informed consent for publication of their clinical details and/or clinical images was obtained from the patient/parent/guardian/relative of the patient. A copy of the consent form is available for review by the Editor of this journal.

\section{Availability of data and material:}

The data that support the findings of this study are available from the corresponding author upon reasonable request.

\section{Competing interests:}

The authors have no competing interest.

\section{Funding:}

Serrapilheira Institute, D’Or Institute for Research and Education, CNPq and CAPES.

\section{Authors' contributions:}

Souza BSF and Rocco PR: conception and design, provision of study material, data analysis and interpretation, manuscript writing, financial support, final approval of the manuscript. Gobatto ALN, Passos RH performed the cell infusions, clinical evaluation, data collection and analysis. Martins GLS and Pinheiro PCG: data collection and analysis. Silva KN, Paredes BD, França LSA and Nonaka CKV: cell manufacturing, characterization and product quality control. Cruz FF and Castro-Faria-Neto HC: Luminex experiments and cytokine analysis. Andrade BB, Barreto-Duarte B, Araújo-Pereira M and Santos RTT: flow cytometry evaluation, data analysis and interpretation. 
Acknowledgements:

This study was supported by grants from Serrapilheira Foundation, D'Or Institute for Research and Education (IDOR), CNPq and CAPES. The authors thank Ms. Alice Andrade for technical support in sample processing and handling. We also thank the healthcare team at the ICU of São Rafael Hospital for the support.

\section{References}

1. Azoulay E, Zafrani L, Mirouse A, Lengliné E, Darmon M, Chevret S. Clinical phenotypes of critically ill COVID-19 patients. Intensive Care Med. 2020 Aug;46(8):1651-1652.

2. Velavan TP, Meyer CG. The COVID-19 epidemic. Trop Med Int Health. 2020 Mar;25(3):278-280.

3. Robba C, Battaglini D, Ball L, Patroniti N, Loconte M, Brunetti I, Vena A, Giacobbe DR, Bassetti M, Rocco PRM, Pelosi P. Distinct phenotypes require distinct respiratory management strategies in severe COVID-19. Respir Physiol Neurobiol. 2020 Aug;279:103455.

4. Zhou F, Yu T, Du R, Fan G, Liu Y, Liu Z, Xiang J, Wang Y, Song B, Gu X, Guan L, Wei Y, Li H, Wu X, Xu J, Tu S, Zhang Y, Chen H, Cao B. Clinical course and risk factors for mortality of adult inpatients with COVID-19 in Wuhan, China: a retrospective cohort study. Lancet. 2020 Mar 28;395(10229):10541062.

5. Armstrong RA, Kane AD, Cook TM. Outcomes from intensive care in patients with COVID-19: a systematic review and meta-analysis of observational studies. Anaesthesia. 2020 Oct;75(10):13401349

6. RECOVERY Collaborative Group, Horby P, Lim WS, Emberson JR, Mafham M, Bell JL, Linsell L, et al. Dexamethasone in Hospitalized Patients with Covid-19 - Preliminary Report. N Engl J Med. 2020 Jul 17:NEJMoa2021436.

7. Cacciapaglia G, Cot C, Sannino F. Second wave COVID-19 pandemics in Europe: a temporal playbook. Sci Rep. 2020 Sep 23;10(1):15514.

8. Khoury M, Cuenca J, Cruz FF, Figueroa FE, Rocco PRM, Weiss DJ. Current status of cell-based therapies for respiratory virus infections: applicability to COVID-19. Eur Respir J. 2020 Jun 4;55(6):2000858.

9. Leng Z, Zhu R, Hou W, Feng Y, Yang Y, Han Q, et al. Transplantation of ACE2-Mesenchymal Stem Cells Improves the Outcome of Patients with COVID-19 Pneumonia. Aging Dis. 2020 Mar 9;11(2):216-228.

10. Tang L, Jiang Y, Zhu M, Chen L, Zhou X, Zhou C, et al. Clinical study using mesenchymal stem cells for the treatment of patients with severe COVID-19. Front Med. 2020 Oct;14(5):664-673.

11. Shu L, Niu C, Li R, Huang T, Wang Y, Huang M, et al. Treatment of severe COVID-19 with human umbilical cord mesenchymal stem cells. Stem Cell Res Ther. 2020 Aug 18;11(1):361.

12. Meng F, Xu R, Wang S, Xu Z, Zhang C, Li Y. Human umbilical cord-derived mesenchymal stem cell therapy in patients with COVID-19: a phase 1 clinical trial. Signal Transduct Target Ther. 2020 Aug 27;5(1):172. 
13. Mazzeo A, Santos EJC. Mesenchymal stem cells in the treatment of coronavirus-induced pneumonia (COVID-19). Einstein (Sao Paulo). 2020;18:eCE5802

14. Peng $H$, Gong $T$, Huang $X$, Sun $X$, Luo $H$, Wang $W$, et al. A synergistic role of convalescent plasma and mesenchymal stem cells in the treatment of severely ill COVID-19 patients: a clinical case report. Stem Cell Res Ther. 2020 Jul 16;11(1):291

15. Liang B, Chen J, Li T, Wu H, Yang W, Li Y, et al. Clinical remission of a critically ill COVID-19 patient treated by human umbilical cord mesenchymal stem cells: A case report. Medicine (Baltimore). 2020 Jul 31;99(31):e21429

16. Zhang Y, Ding J, Ren S, Wang W, Yang Y, Li S, et al. Intravenous infusion of human umbilical cord Wharton's jelly-derived mesenchymal stem cells as a potential treatment for patients with COVID-19 pneumonia. Stem Cell Res Ther. 2020 May 27;11(1):207.

17. Chen X, Shan Y, Wen Y, Sun J, Du H. Mesenchymal stem cell therapy in severe COVID-19: A retrospective study of short-term treatment efficacy and side effects. J Infect. 2020 Oct;81(4):647679.

18. Zhu Y, Zhu R, Liu K, Li X, Chen D, Bai D, et al. Human Umbilical Cord Mesenchymal Stem Cells for Adjuvant Treatment of a Critically III COVID-19 Patient: A Case Report. Infect Drug Resist. 2020 Sep 28;13:3295-3300.

19. COVID-19 Treatment Guidelines Panel. Coronavirus Disease 2019 (COVID-19) Treatment Guidelines. National Institutes of Health. Available at https://www.covid19treatmentguidelines.nih.gov/. Accessed 23 nov 2020 .

20. Mendonça MV, Larocca TF, de Freitas Souza BS, Villarreal CF, Silva LF, Matos AC, et al. Safety and neurological assessments after autologous transplantation of bone marrow mesenchymal stem cells in subjects with chronic spinal cord injury. Stem Cell Res Ther. 2014 Nov 17;5(6):126.

21. Rovira Gonzalez YI, Lynch PJ, Thompson EE, Stultz BG, Hursh DA. In vitro cytokine licensing induces persistent permissive chromatin at the Indoleamine 2,3-dioxygenase promoter. Cytotherapy. 2016 Sep;18(9):1114-28.

22. Moll G, Ankrum JA, Kamhieh-Milz J, Bieback K, Ringdén O, Volk HD, Geissler S, Reinke P. Intravascular Mesenchymal Stromal/Stem Cell Therapy Product Diversification: Time for New Clinical Guidelines. Trends Mol Med. 2019 Feb;25(2):149-163.

23. Dominici M, Le Blanc K, Mueller I, Slaper-Cortenbach I, Marini F, Krause D, et al. Minimal criteria for defining multipotent mesenchymal stromal cells. The International Society for Cellular Therapy position statement. Cytotherapy. 2006;8(4):315-7.

24. Sánchez-Guijo F, García-Arranz M, López-Parra M, Monedero P, Mata-Martínez C, Santos A, et al. Adipose-derived mesenchymal stromal cells for the treatment of patients with severe SARS-CoV-2 pneumonia requiring mechanical ventilation. A proof of concept study. EClinicalMedicine. 2020 Aug;25:100454.

25. Guo Z, Chen Y, Luo X, He X, Zhang Y, Wang J. Administration of umbilical cord mesenchymal stem cells in patients with severe COVID-19 pneumonia. Crit Care. 2020 Jul 11;24(1):420. 
26. Sengupta V, Sengupta S, Lazo A, Woods P, Nolan A, Bremer N. Exosomes Derived from Bone Marrow Mesenchymal Stem Cells as Treatment for Severe COVID-19. Stem Cells Dev. 2020 Jun 15;29(12):747-754.

27. Gu SX, Tyagi T, Jain K, Gu VW, Lee SH, Hwa JM, et al. Thrombocytopathy and endotheliopathy: crucial contributors to COVID-19 thromboinflammation. Nat Rev Cardiol. 2020 Nov 19:1-16.

28. Hadjadj J, Yatim N, Barnabei L, Corneau A, Boussier J, Smith N, et al. Impaired type I interferon activity and inflammatory responses in severe COVID-19 patients. Science. 2020 Aug 7;369(6504):718-724.

29. Huang C, Wang Y, Li X, Ren L, Zhao J, et al. Clinical features of patients infected with 2019 novel coronavirus in Wuhan, China. Lancet. 2020 Feb 15;395(10223):497-506.

30. Kyriazopoulou E, Leventogiannis K, Norrby-Teglund A, Dimopoulos G, Pantazi A, Orfanos SE, et al. Macrophage activation-like syndrome: an immunological entity associated with rapid progression to death in sepsis. BMC Med. 2017 Sep 18;15(1):172.

31. Giamarellos-Bourboulis EJ, Netea MG, Rovina N, Akinosoglou K, Antoniadou A, Antonakos N, Damoraki G, et al. Complex Immune Dysregulation in COVID-19 Patients with Severe Respiratory Failure. Cell Host Microbe. 2020 Jun 10;27(6):992-1000.e3.

32. van der Poll T, van de Veerdonk FL, Scicluna BP, Netea MG. The immunopathology of sepsis and potential therapeutic targets. Nat Rev Immunol. 2017 Jul;17(7):407-420.

33. Shin JY, Wang CY, Lin CC, Chu CL. A recently described type 2 conventional dendritic cell (cDC2) subset mediates inflammation. Cell Mol Immunol. 2020 Jul 30.

34. Pyle CJ, Uwadiae FI, Swieboda DP, Harker JA. Early IL-6 signalling promotes IL-27 dependent maturation of regulatory $\mathrm{T}$ cells in the lungs and resolution of viral immunopathology. PLoS Pathog. 2017 Sep 27;13(9):e1006640.

35. Auffray C, Fogg D, Garfa M, Elain G, Join-Lambert O, Kayal S, et al. Monitoring of blood vessels and tissues by a population of monocytes with patrolling behavior. Science. 2007 Aug 3;317(5838):66670.

36. Jakubzick C, Tacke F, Ginhoux F, Wagers AJ, van Rooijen N, Mack M, Merad M, Randolph GJ. Blood monocyte subsets differentially give rise to CD103+ and CD103-pulmonary dendritic cell populations. J Immunol. 2008 Mar 1;180(5):3019-27.

37. Cros J, Cagnard N, Woollard K, Patey N, Zhang SY, Senechal B, et al. Human CD14dim monocytes patrol and sense nucleic acids and viruses via TLR7 and TLR8 receptors. Immunity. 2010 Sep 24;33(3):375-86.

38. Carlin LM, Stamatiades EG, Auffray C, Hanna RN, Glover L, Vizcay-Barrena G, et al. Nr4a1-dependent Ly6C(low) monocytes monitor endothelial cells and orchestrate their disposal. Cell. 2013 Apr 11;153(2):362-75.

39. Caricchio R, Gallucci M, Dass C, Zhang X, Gallucci S, Fleece D, Bromberg M, Criner GJ; Temple University COVID-19 Research Group. Preliminary predictive criteria for COVID-19 cytokine storm. Ann Rheum Dis. 2020 Sep 25:annrheumdis-2020-218323. 
40. Sun X, Wang T, Cai D, Hu Z, Chen J, Liao H, Zhi L, Wei H, Zhang Z, Qiu Y, Wang J, Wang A. Cytokine storm intervention in the early stages of COVID-19 pneumonia. Cytokine Growth Factor Rev. 2020 Jun;53:38-42.

\section{Figures}

A

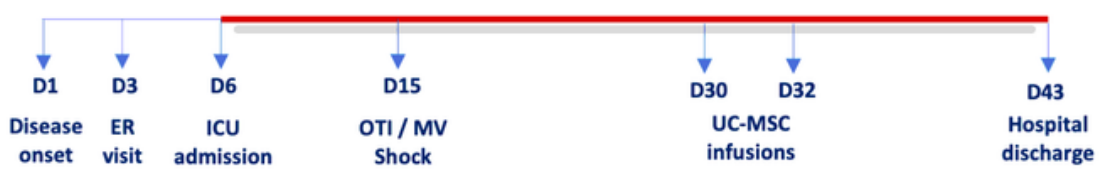

B
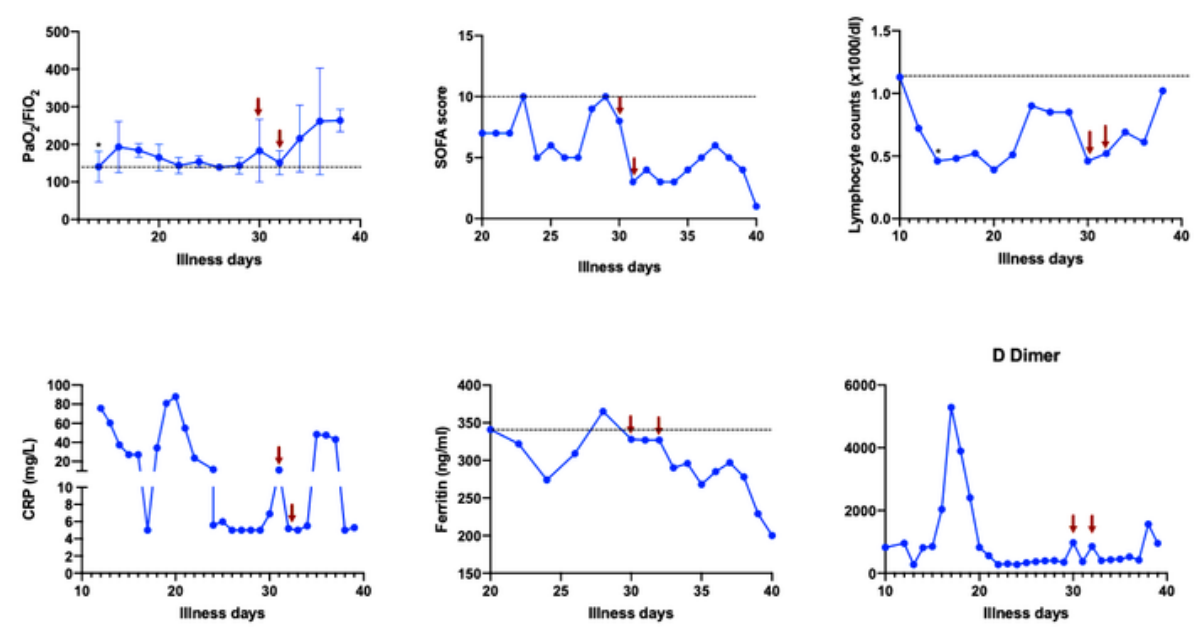

C
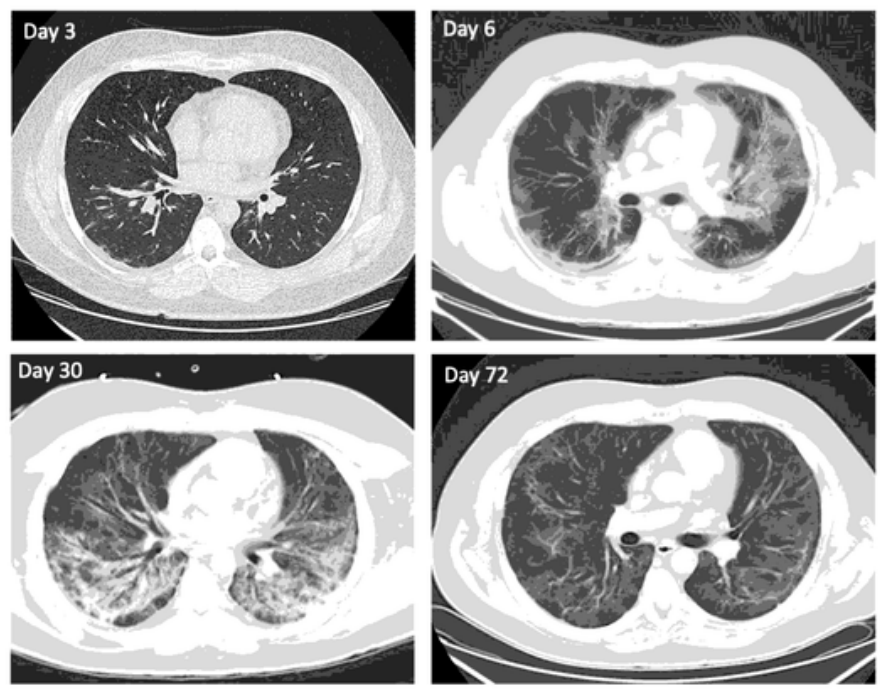
Evolution of clinical, laboratory and radiological parameters. (A) Timeline of the duration of patient stay in the intensive care unit. OTI: orotracheal intubation; MV: mechanical ventilation; UC-MSC: umbilical cord-derived mesenchymal stromal cells. Arrows represent the time of MSC infusions; respective samples were collected prior MSC infusion. * = day of orotracheal intubation. (B) Data are represented as single values or mean $\pm S D$ of the values of different measurements performed at each day. (C) Representative images of chest CT scan performed at different timepoints. (D) Al-based quantification of lung injury.

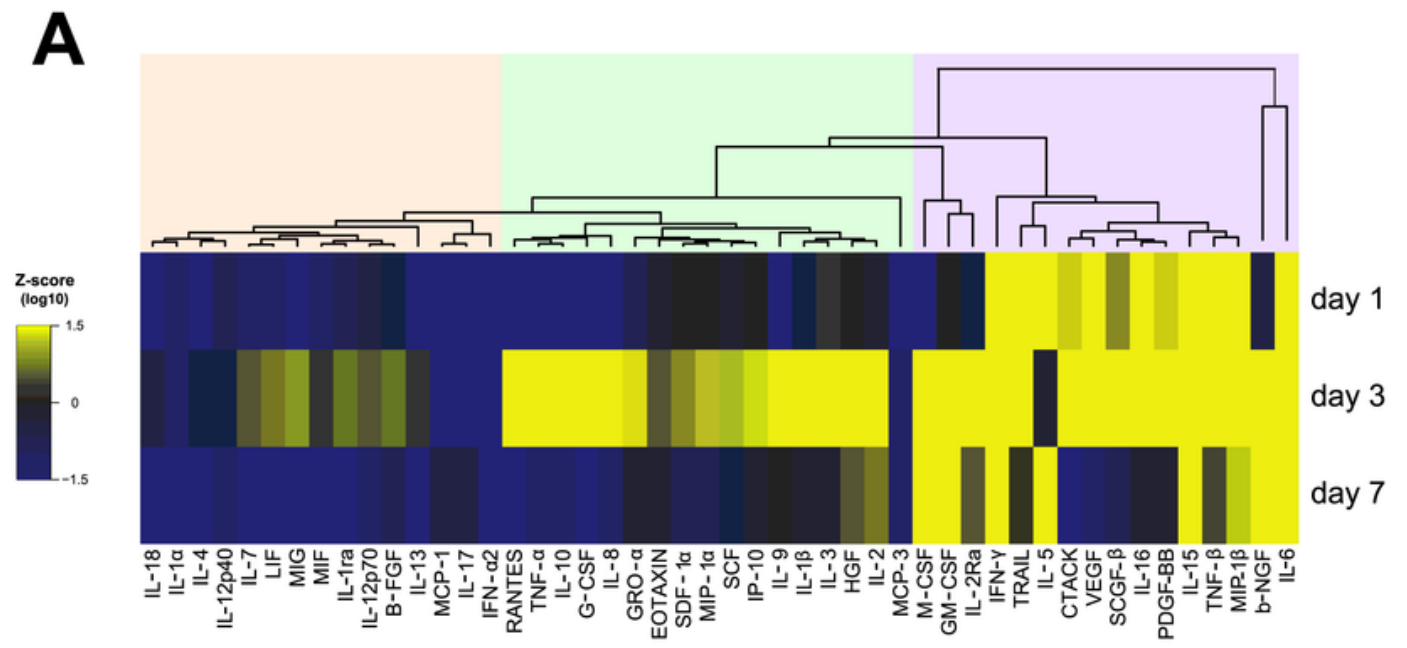

B

Calcineurin-regulated NFAT-dependent transcription in lymphocytes

SHP2 signaling

AP-1 transcription factor network

Glucocorticoid receptor regulatory network

IL27-mediated signaling events

Calcium signaling in the CD4+ TCR pathway

IL23-mediated signaling events

Calcineurin-regulated NFAT-dependent transcription in lymphocytes

IL12 signaling mediated by STAT4

IL12-mediated signaling events

Glucocorticoid receptor regulatory network

Cellular roles of Anthrax toxin

C

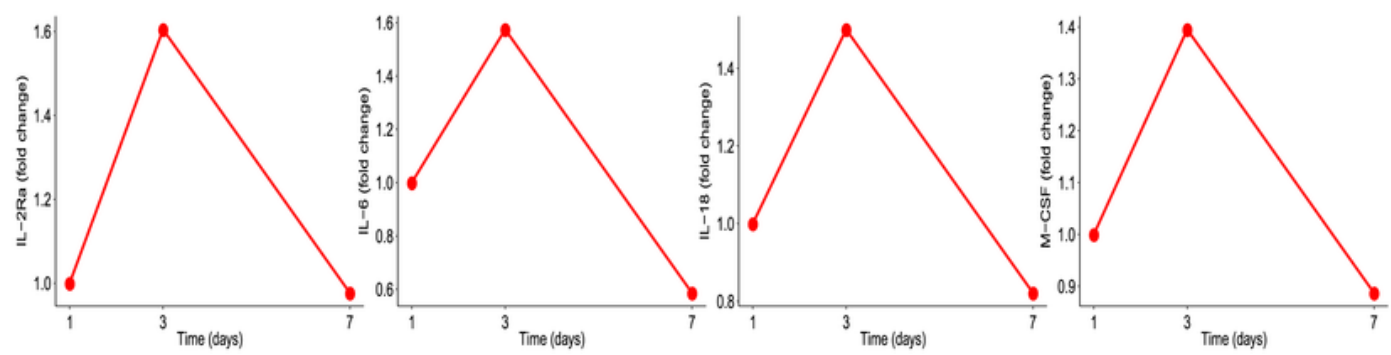

Figure 2 
Expression profile of cytokines, chemokines and growth factors by Luminex assay. (A) A Heatmap was designed to depict the overall profile of biomarkers among times, according to log10 of Mean Fluorescent Intensity (MFI). A one-way hierarchical cluster analysis (Ward's method) was performed. Dendrograms represent Euclidean distance. (B) Enrichment analysis for each cluster at NCI Nature database. Top 4 of pathways are show, ranked by $p$ value. (C) IL-2Ra, IL-6, IL-18 and M-CSF showed a higher fold-change (+-0.5) of MFI over time. Samples were collected on the day of the 1st MSC infusion (D1, pre-infusion), and subsequent days (D3 and D7).
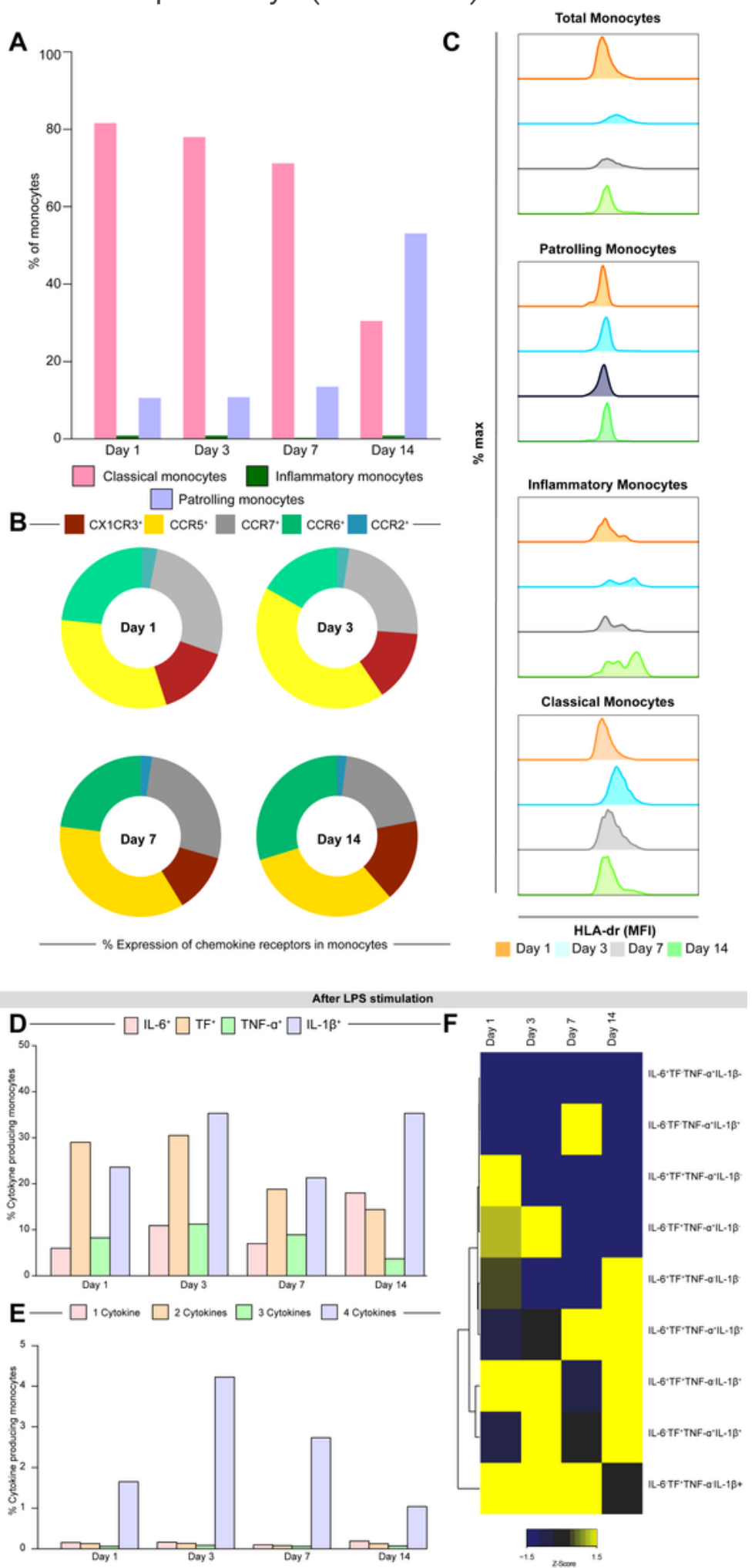


\section{Figure 3}

Classical monocytes (CD14++CD16-) are more prevalent up to 7 days after treatment initiation. (A) The frequency of classical (CD14++CD16-), inflammatory (CD14++CD16+) and patrolling (CD14+CD16+) monocytes (pink, green and purple, respectively) exhibits the same overall profile until day 7. On day 14, there is an increase in patrolling monocytes frequency, that exceeds $50 \%$ of all monocyte populations. (B) Observing the frequencies, we notice that CCR5 and CCR7 are the most prevalent markers over time. (C) Median fluorescence intensity of HLD-DR among monocytes subpopulation over period post treatment. Assessment of monocyte polyfunctionality following overnight LPS stimulation. (A) The frequency of IL6, TF, TNF-a and IL-1B expression is shown. (B) The frequencies of cytokine producer's subpopulations are shown. (C) A Heatmap was designed to depict the overall profile of cytokine producer subpopulation of monocytes over times. A one-way hierarchical cluster analysis (Ward's method) was performed. Dendrograms represent Euclidean distance.
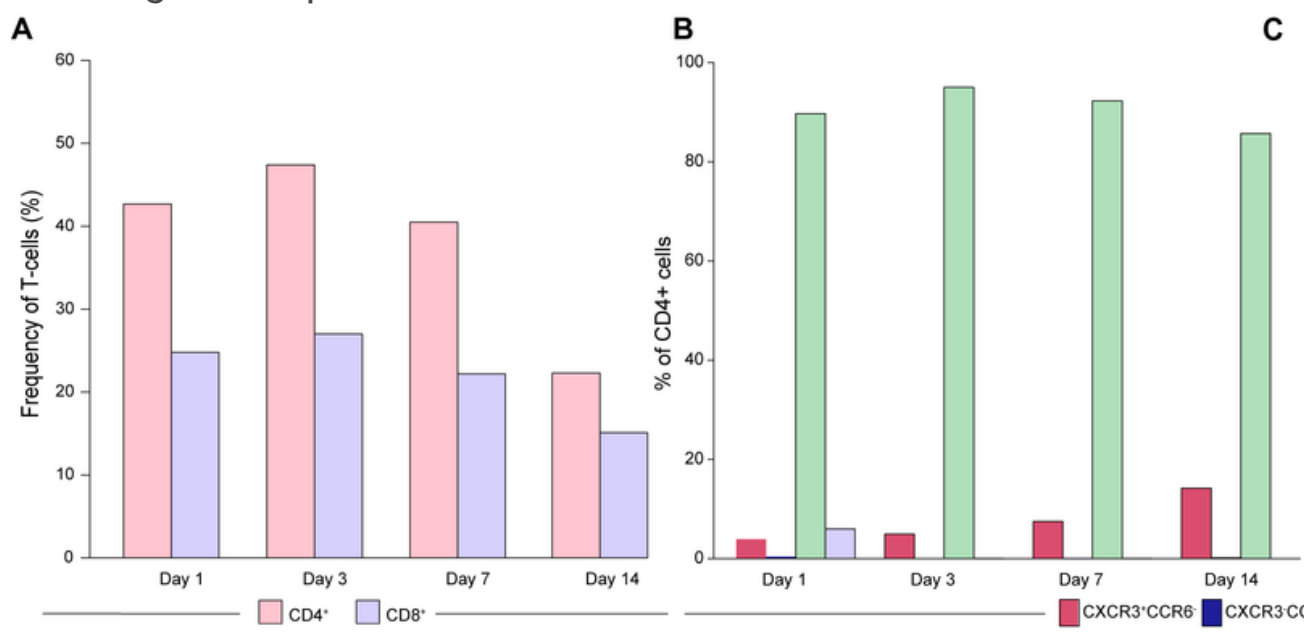

C

D
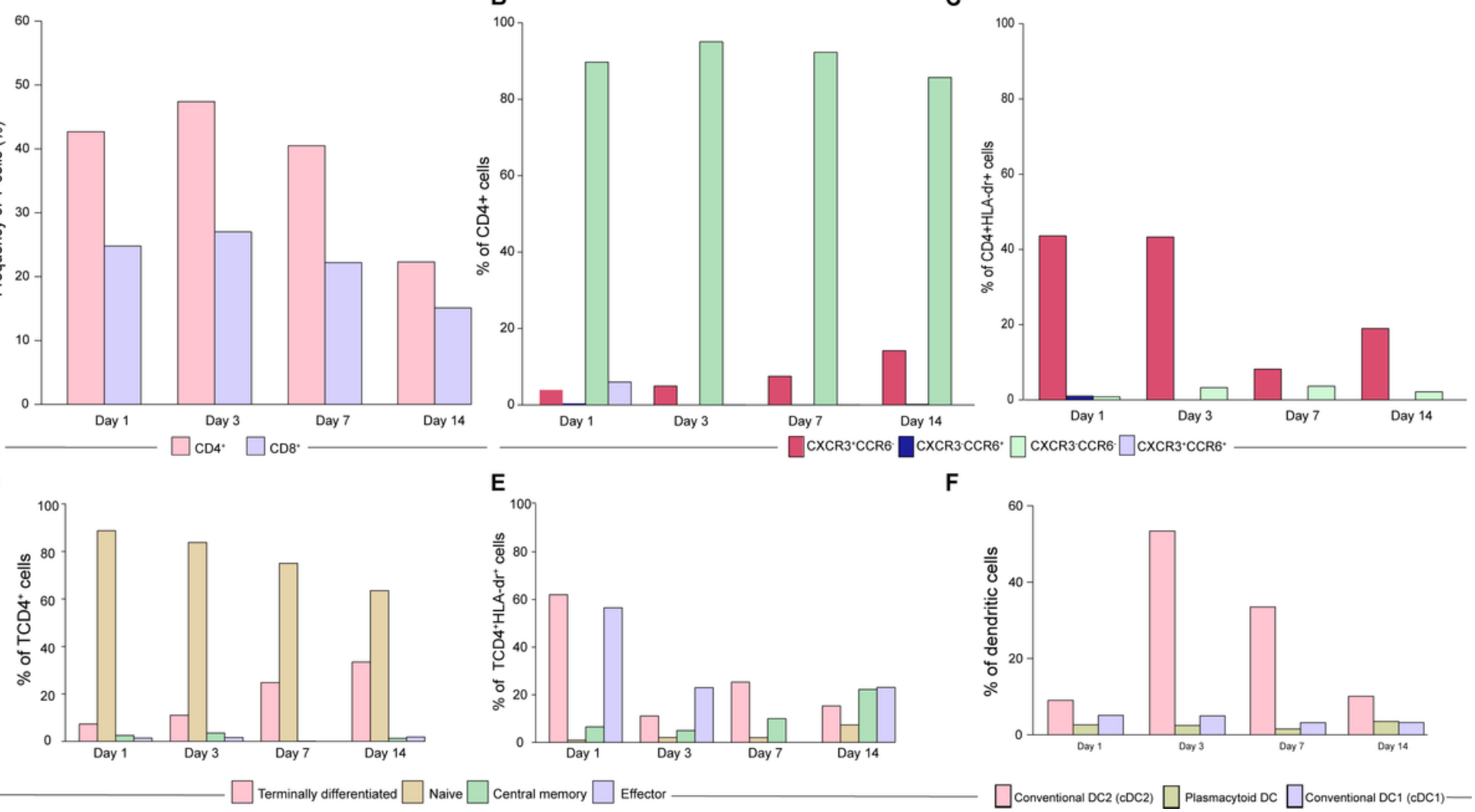

$\square$ Conventional DC2 (cDC2) $\square$ Plasmacytoid DC

\section{Figure 4}

Dynamics of CD4+ T helper subsets turnover following treatment initiation. (A) . Frequencies of CD4+ and CD8+ T Lymphocytes over time. (B) The differential expression of chemokine receptors CCR6 and CXCR3 in CD4 T cells are shown, further indicating the frequencies of Th1 (CXCR3+CCR6-), Th1 Star (CXCR3+CCR6+), Th2 (CXCR3-CCR6-), and Th17 (CXCR3-CCR6+) subpopulations. (C) The differential expression of chemokine receptors CCR6 and CXCR3 in activated CD4 T cells are shown, further indicating the frequencies of Th1 (CXCR3+CCR6-), Th1 Star (CXCR3+CCR6+), Th2 (CXCR3-CCR6-), and Th17 (CXCR3-CCR6+) subpopulations. Figure 5. Dynamics of memory CD4+ T cells subsets turnover 
following treatment initiation. (A) The frequencies of naïve (CD45+CD27+), central memory (CD45CD27+), effector (CD45-CD27-) and terminally differentiated cells (CD45+CD27-) are shown, without activation. (B) The frequencies of naïve (CD45+CD27+), central memory (CD45-CD27+), effector (CD45CD27-), and terminally differentiated cells (CD45-CD27+) are shown in activated (HLA-DR+) cells. Conventional dendritic cells subtype 2 ( $C D C 2)$ frequencies increased on day 3 . The frequency of conventional DC2 (HLA-dr+CD11c+CD141-), plasmacytoid DCs (HLA-dr+CD11c-CD123+), and conventional DC1 (HLA-dr+CD11c+CD141+) (pink, brown and light purple, respectively) are shown.

\section{Supplementary Files}

This is a list of supplementary files associated with this preprint. Click to download.

- SupplementalFigure1.tif

- SupplementalFigure2.tif

- SupplementalTable.docx 